\title{
Electrohydrodynamic Direct-Write Orderly Micro/Nanofibrous Structure on Flexible Insulating Substrate
}

\author{
Jiang-Yi Zheng, Hai-Yan Liu, Xiang Wang, Yang Zhao, Wei-Wei Huang, \\ Gao-Feng Zheng, and Dao-Heng Sun \\ Department of Mechanical and Electrical Engineering, Xiamen University, Xiamen 361005, China \\ Correspondence should be addressed to Gao-Feng Zheng; 65404580@qq.com
}

Received 13 February 2014; Revised 5 May 2014; Accepted 5 May 2014; Published 26 May 2014

Academic Editor: Huarong Nie

Copyright (c) 2014 Jiang-Yi Zheng et al. This is an open access article distributed under the Creative Commons Attribution License, which permits unrestricted use, distribution, and reproduction in any medium, provided the original work is properly cited.

\begin{abstract}
AC pulse-modulated electrohydrodynamic direct-writing (EDW) was utilized to direct-write orderly micro/nanofibrous structure on the flexible insulating polyethylene terephthalate (PET) substrate. During the EDW process, AC electrical field induced charges to reciprocate along the jet and decreased the charge repulsive force that applied on charged jet. Thanks to the smaller charge repulsive force, stable straight jet can be built up to direct-write orderly micro/nanofibrous structures on the insulating substrate. The minimum motion velocity required to direct-write straight line fibrous structure on insulating PET substrate was $700 \mathrm{~mm} / \mathrm{s}$. Moreover, the influences of AC voltage amplitude, frequency, and duty cycle ratio on the line width of fibrous structures were investigated. This work proposes a novel solution to overcome the inherent charge repulsion emerging on the insulating substrate, and promotes the application of EDW technology on the flexible electronics.
\end{abstract}

\section{Introduction}

With the unique advantages, flexible electronics attracts significant interest from all over the world and has wide application prospects in the fields of information, energy, health, microsensors, and national defense [1-3]. To date, the functional patterns on flexible electronics can be deposited by sputter coating [4], inkjet printing [5], and airbrush spraying [6]. Thanks to features of low cost, low waste, noncontact, and good materials compatibility, inkjet printing has been regarded as one of the most effective technologies to realize the large-scale manufacturing of flexible electronic devices without stencil plate and etching process $[7,8]$.

Electrohydrodynamic direct-write (EDW) technique is a novel inkjet technology based on the principle of nearfield electrospinning (NFES) [9], in which the stable straight charged jet is used to fabricate orderly micro/nanostructure. EDW used electrical field to drive charged jet injection from the tip of Taylor cone below the spinneret, and the diameter of charged jet is significantly smaller than the inner diameter of the spinneret. At present, EDW technology has displayed great application potential in many fields. Chang et al.
[10] direct-write straight PVDF nanofiber with diameter of $500 \mathrm{~nm} \sim 6.5 \mu \mathrm{m}$ to fabricate high energy polymeric nanogenerator. Wang et al. [11] direct-write $\mathrm{ZnO}$ nanofibers to fabricate field-effect transistor. Kim et al. [12] used EDW to fabricate $\mathrm{TiO}_{2}$ nanofibers as ultrasensitive chemiresistors.

However, there are still some problems that prevent EDW technique for industrial applications. A critical one is that EDW on insulating substrate, especially flexible polymer substrate such as polyethylene terephthalate (PET) and polyimide (PI), is still a challenge. DC electrical field is used as the energy source to stretch viscoelastic solution in commonly during EDW process, by which charged jet can be ejected from the tip of Taylor cone [13]. Liquid jet also carried away the charge from the spinneret. As the liquid jet is deposited on the collector, charges are also transferred to collector. The charges accumulated on collector surface change the electrical field distribution and provide Coulomb repulsive force on the following jet. Owing to the short distance between spinneret and collector, Coulomb repulsive force from accumulated charges plays a key role in the deposition behaviors of EDW jet [14]. The polymer substrate, for example, PET substrate, has low conductivity and large charge 
relaxation time. The accumulated charges on PET substrate require longer relaxation time to leak from polymer substrate to ground. On the other hand, polymer substrate would be polarized by the electrical field and would provide an excess of repulsive force on the following charged jet. The large Coulomb repulsive force blocks the stable ejection and pattern deposition of EDW charged jet on polymer substrate. Polymer thin film is one of the multipurpose flexible basements for flexible electronics. At present, direct-writing of orderly micro/nanostructures on flexible polymer substrate have become the key for the application research of EDW technology.

Alternating electrical field changes the charge transfer characteristics in the charged liquid jet and provides a good way to reduce the interferences from charge repulsion $[15,16]$. AC electrical field also induces the charge transfer along the surface of insulate substrate and prevents polarization inside the insulating substrate [17]. Alternating electrical field may be a good method to promote the application of EDW technology on the flexible electronics.

In this paper, AC pulse voltage is introduced to study the deposition behaviors of EDW charged jet on the PET substrate. The effects of AC electrical fields on the rheology and injection process of viscoelastic solution were also investigated. And then, orderly nanofiber can be direct-written on the PET substrate under AC electrical field.

\section{Experiment Details}

The schematic diagram of EDW apparatus was illustrated in Figure 1, where a high AC power source (DW-P503LACDE, China) and a high DC power source (DW-P403$1 \mathrm{AC}, \mathrm{China}$ ) were used, respectively, to provide electrical field for the ejection of EDW jet. The anode of power source was connected to the stainless nozzle and the cathode was connected to a grounded PET substrate. The grounded PET substrate (the insert in Figure 1(a)) was fixed on a motion platform (Parker Linear Motor, USA), which consisted of an EDW module and a control module. The maximum velocity of linear motor was $5 \mathrm{~m} / \mathrm{s}$; the motion track and velocity of collector can be controlled by the host computer. Stainless nozzle (inner diameter was $210 \mu \mathrm{m}$ and outer diameter was $400 \mu \mathrm{m})$ was used as spinneret and the distance between spinneret and collector was in the range of $2 \sim 10 \mathrm{~mm}$. Polymer solution was continuously delivered to the spinneret by a syringe pump (Harvard 11 Pico Plus, USA) at a flow rate of $50 \mu \mathrm{L} / \mathrm{hr}$. A CCD camera (Sony SSC-DC80, Japan) and a high speed camera (GX-1, Japan) were used to observe the ejection and deposition process. Polyethylene oxide (PEO, $\mathrm{M}_{W}=300,000 \mathrm{~g} / \mathrm{mol}$ ) solution in a mixed solvent of water and ethanol $(v: v=3: 1)$ was used as an experimental material. Before the experiments, the PET substrate was cleaned in deionized water and acetone and then dried by a hot air blower.

Figure 1(b) demonstrates the typical waveform of AC pulse voltage applied in the present work. The morphology of patterned micro/nanostructures was examined by using an optical microscope (Mitutoyo, Japan) and a scanning electron microscope (LEO 1530 Gemini, Germany).

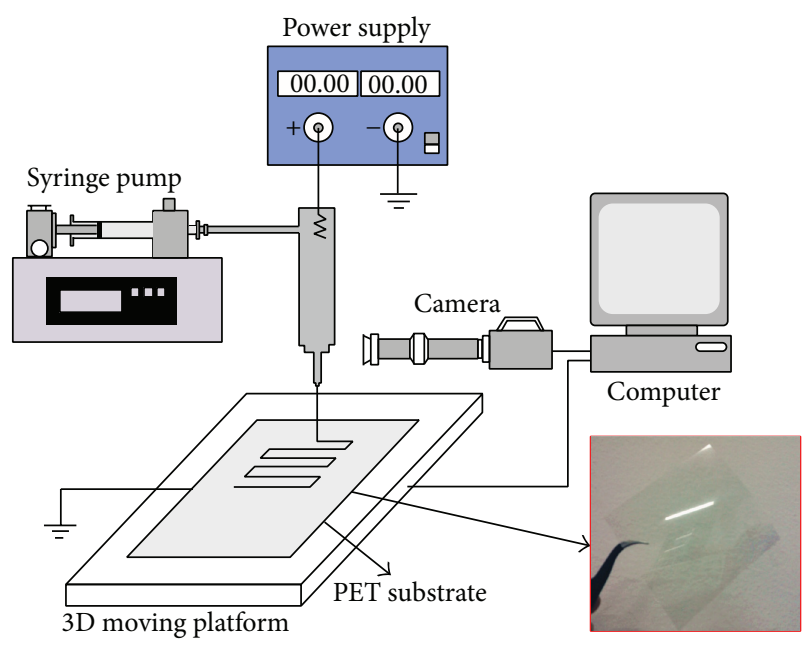

(a)

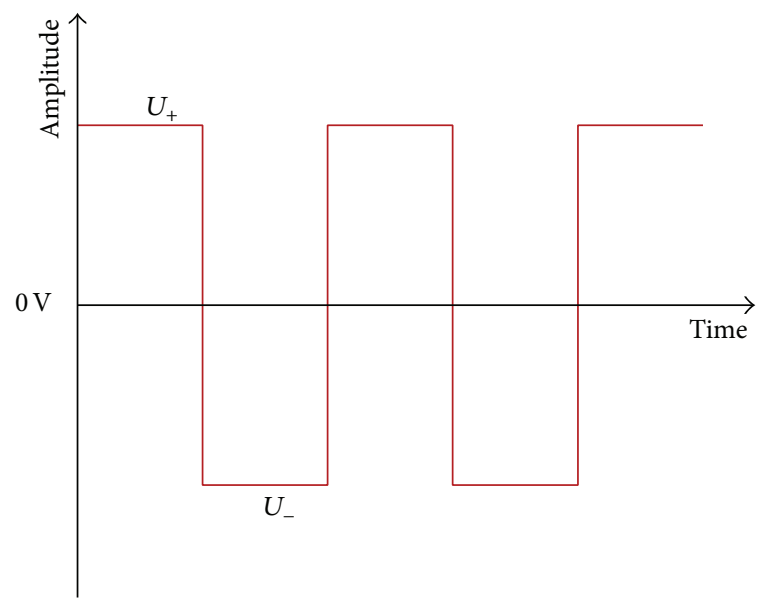

(b)

FIgURE 1: (a) The schematic diagram of EDW experimental setup; (b) wave shape of AC pulse voltage.

\section{Results and Discussion}

Firstly, nanofibers deposited on a fixed insulating PET substrate under AC and DC electrical fields were investigated. In these experiments, the concentration of PEO solution $C$, the distance between spinneret and insulated substrate $H$, and the solution supply rate $Q$ were $14 \mathrm{wt} \%, 10 \mathrm{~mm}$, and $50 \mu \mathrm{L} / \mathrm{hr}$, respectively.

It is difficult to direct-write patterns on insulating substrate, for example, PET substrate, by using EDW with DC electrical field applied. Figure 2(a) shows nanofibers collected on the PET substrate under a constant DC voltage of $4 \mathrm{kV}$. During EDW process, the liquid jet ejected from Taylor cone under the electrical field force. When the jet is deposited on substrate, charge carried by the liquid would leak through substrate to ground. Owing to the insulating PET substrate and lower charge leakage rate and large charge relaxation time, more charges would accumulate on the substrate and apply Coulomb repulsive force on the following jet. Due to the 


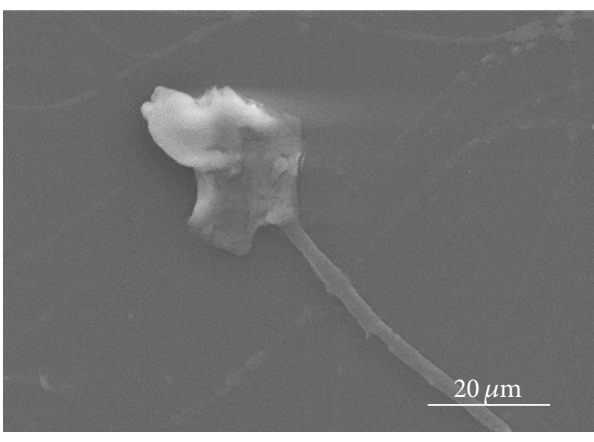

(a)

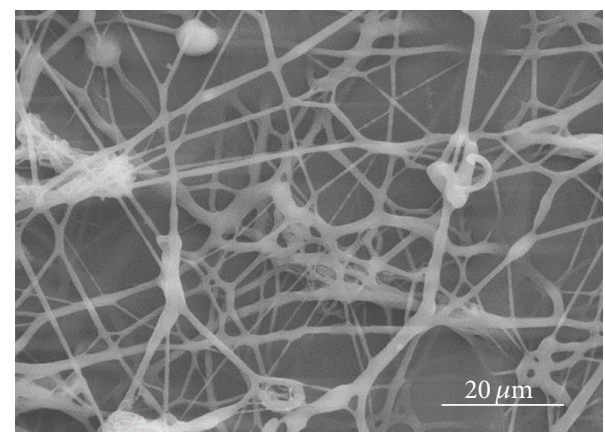

(b)

FIGURE 2: Micro/nanostructures deposited on PET insulate substrate under DC and AC electrical fields. (a) Nanofibers deposited on the PET substrate under the DC electrical field, experimental condition: $U=4 \mathrm{kV}, H=10 \mathrm{~mm}, C=14 \mathrm{wt} \%$, and $Q=50 \mu \mathrm{L} / \mathrm{hr}$; (b) nanofibers deposited on the PET substrate under the AC electrical field, experimental condition: $U_{-}=-4 \mathrm{kV}, U_{+}=4 \mathrm{kV}, f_{\text {app }}=500 \mathrm{~Hz}, \rho=50 \%$, $H=10 \mathrm{~mm}, C=14 \mathrm{wt} \%$, and $Q=50 \mu \mathrm{L} / \mathrm{hr}$.

Coulomb repulsive force, charged jets are repelled from deposition zone below spinneret. Therefore, fewer nanofibers can be deposited on the polymer substrate. By applying AC electrical field, we successfully obtained PEO nanofibers on the PET substrate, as shown in Figure 2(b). The voltage frequency $f_{\text {app }}$, duty cycle ratio $\rho$, negative amplitude $U_{-}$, and positive amplitude $U_{+}$of AC power source were $500 \mathrm{~Hz}, 50 \%,-4 \mathrm{kV}$, and $4 \mathrm{kV}$, respectively. Contrary to the DC electrical field applied, there were plenty of nanofibers generated on the substrate just below the spinneret. AC electrical field changes the charge transfer characteristics in the charged jet and reduces the Coulomb repulsive force from the residual charges that accumulated on deposited nanofiber and PET substrate. Thus more nanofibers can be collected on PET under AC electrical field. The collection time of EDW nanofibers for each sample shown in Figure 2 was $10 \mathrm{~s}$.

A stable straight jet is beneficial for the direct-writing of orderly micro/nanostructures. Because of the short distance between spinneret and substrate in EDW, the residual charge accumulated on the insulating substrate would play an important role on the motion deposition process of charged jet. In the applied DC electrical field case, the Coulomb repulsion force, which stemmed from the residual charge accumulated on PET substrate, made the jet deviate from the desired straight motion track, as shown in Figure 3(a). The fact that charged jet cannot be deposited on the PET substrate under the DC electrical field hinders the potential applications of EDW technology on flexible electronic manufacturing.

Due to the large Coulomb repulsive force from the insulating substrate, stable jet cannot be built up to direct-write uniform structures. Figure 3(b) shows the "direct-written" micro/nanostructures under DC electrical field. These fibrous structures were randomly oriented on the PET substrate and the diameter had a large distribution range due to the larger repulsive force and unstable stretching. In EDW process, with the short distance between spinneret and collector, the charged jet was repelled by the deposited ones and surpassed the constraint of the jet inner stress.

The alternating of positive and negative voltages would induce charge transferred upward and downward along the jet, which reduced the Coulomb repulsive force applied on the following jet. With the help of AC electrical field, stable straight jet between spinneret and collector can be generated to direct-write continuous uniform micro/nanofibrous structures. The alternating polarity which stemmed from AC electrical field reduces the inherent instability of electrospinning jet $[18,19]$ and provides an excellent way to increase the stability of charged jet between the spinneret and insulating substrate. Figure 3(c) shows that the charged jet ejected from the spinneret kept in a straight track without splitting and whipping, under an AC electrical field. The voltage frequency $f_{\text {app }}$, duty cycle ratio $\rho$, negative amplitude $U_{-}$, and positive amplitude $U_{+}$of AC power source used in the experimentwere $500 \mathrm{~Hz}, 50 \%,-2.3 \mathrm{kV}$, and $2.3 \mathrm{kV}$, respectively. The direct-written uniform fibrous structure can be direct-written as spiral pattern along the motion track of collector, as shown in Figure 3(d).

The deposition behaviors of charged jet on the insulating substrate were further investigated. As discussed in previous works, the velocity of substrate $v_{\text {col }}$ was a key factor that defined the deposition and the diameter of direct-written fibrous structures $[13,20]$. As shown in Figure 4, when motion velocity of substrate $v_{\text {col }}$ increased from $50 \mathrm{~mm} / \mathrm{s}$ to $1500 \mathrm{~mm} / \mathrm{s}$, the deposition pattern of direct-written fibrous structure changed from waved shape to straight line structure. The negative amplitude $U_{-}$and positive amplitude $U_{+}$ of $\mathrm{AC}$ power source were $-4 \mathrm{kV}$ and $4 \mathrm{kV}$, respectively. The motion track and velocity of collector were controlled by the host computer and the distance between parallel lines in the direct-written pattern was $70 \mu \mathrm{m}$. When motion velocity of substrate $v_{\text {col }}$ was larger than $700 \mathrm{~mm} / \mathrm{s}$, the whipping of charged jet can be overcome by the stretching force that stemmed from motion substrate. Due to the Coulomb repulsive force, the minimum motion velocity required to direct-write straight line fibrous structure on insulating PET substrate was larger than that on conductive or semiconductive substrate [21].

To obtain stable charged jet in EDW, proper parameters of AC electrical field including the voltage amplitude, voltage frequency, and duty cycle ratio were required and the effect 


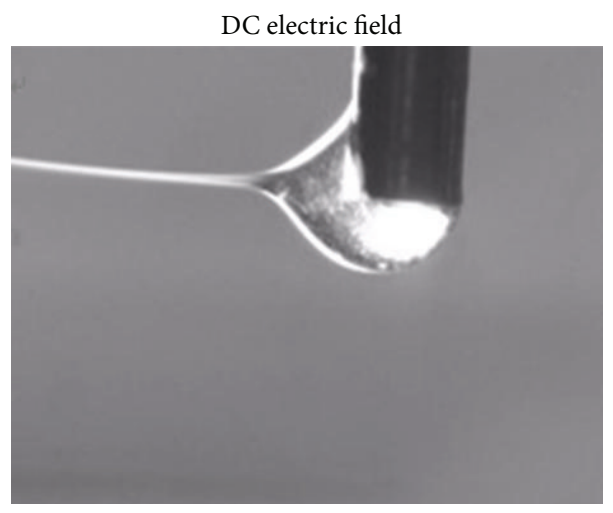

(a)

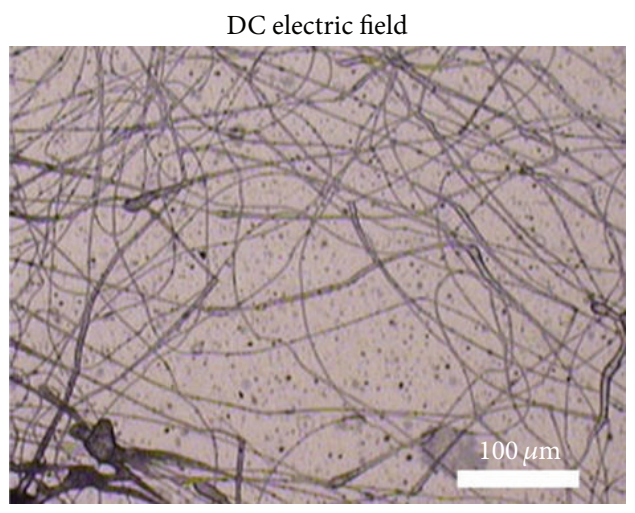

(b)

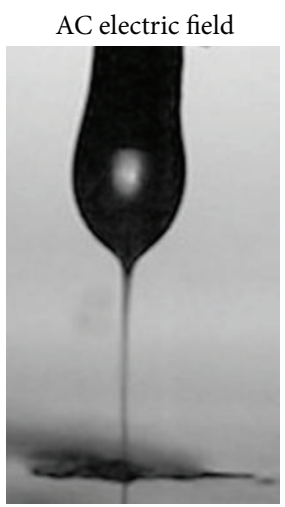

(c)

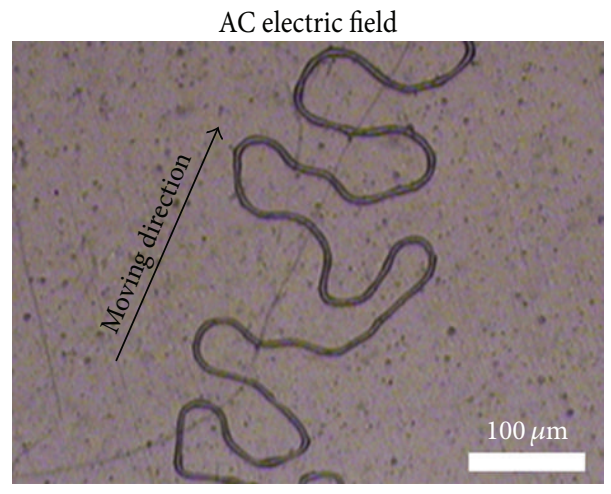

(d)

FIGURE 3: (a) Charged jet deviates from the straight motion track under DC electrical field; (b) nonuniform fibrous structure deposited on insulating PET substrate under DC electrical field. Experimental condition: $U=3 \mathrm{kV}, H=2 \mathrm{~mm}, \mathrm{Q}=50 \mu \mathrm{L} / \mathrm{hr}$, and $C=14 \mathrm{wt} \%$; (c) stable straight jet under AC electrical field on the insulating substrate; (d) uniform fibrous structure direct-written on insulating PET substrate. Experimental condition: $C=14 \mathrm{wt} \%, U_{-}=-2.3 \mathrm{kV}, U_{+}=2.3 \mathrm{kV}, f_{\text {app }}=500 \mathrm{~Hz}, \rho=50 \%, H=2 \mathrm{~mm}, Q=50 \mu \mathrm{L} / \mathrm{hr}$, and $v_{\text {col }}=5 \mathrm{~mm} / \mathrm{s}$.

of these parameters was also investigated further. To study the effect of AC voltage on the EDW patterns, we fixed the positive amplitude $U_{+}$at $3 \mathrm{kV}$ and performed various negative amplitudes while the other parameters were set as $v_{\text {col }}=$ $20 \mathrm{~mm} / \mathrm{s}, H=2 \mathrm{~mm}, f_{\text {app }}=750 \mathrm{~Hz}, \delta=50 \%$, and $Q=$ $50 \mu \mathrm{L} / \mathrm{hr}$. Figure 5 shows that the average line width of EDW patterns increased with the increasing of negative voltage amplitude and reached its maximum value when the negative voltage amplitude equals the positive ones. The opposite trend appeared as the negative voltage amplitude succeeding the positive ones. The amount of free charge on the jet depends on the applied voltage in EDW. When the negative voltage amplitude equals the positive voltage ones, the equivalent charge density and electrical field force reach the minimum value, as the smaller electrical field force and stretch ratio lead to larger line width.

Figure 6 demonstrates the relationship between the AC voltage frequency $f_{\text {app }}$ and the EDW patterns. The experimental parameters were set as $C=14 \mathrm{wt} \%, U_{-}=-1.5 \mathrm{kV}$, $U_{+}=3.5 \mathrm{kV}, \rho=50 \%, H=2 \mathrm{~mm}$, and $Q=50 \mu \mathrm{L} / \mathrm{hr}$. It can be seen that the average line width increased from $18 \mu \mathrm{m}$ to $33 \mu \mathrm{m}$ when increasing the $\mathrm{AC}$ voltage frequency $f_{\text {app }}$ from $0 \mathrm{~Hz}$ to $750 \mathrm{~Hz}$, due to the equivalent electrical field force which decreased with the increase of voltage frequency. Then, line width reached the minimum value when $\mathrm{AC}$ voltage frequency $f_{\text {app }}$ was $750 \mathrm{~Hz}$. When AC voltage frequency $f_{\text {app }}$ was larger than $750 \mathrm{~Hz}$, average line width decreased with the increase of voltage frequency $f_{\text {app }}$. The relaxation time scale of liquid jet was larger than that of electrical field. With the high AC voltage frequency $f_{\text {app}}$, liquid jet did not have enough time to shrink back. Thus, equivalent stretch force increased with the increase of $\mathrm{AC}$ voltage frequency $f_{\text {app }}$, which helped to decrease the line width.

Figure 7 illustrates the effect of duty cycle ratio on the line width of EDW fibrous patterns. The experimental parameters were set as $C=14 \mathrm{wt} \%, U_{-}=-1.5 \mathrm{kV}, U_{+}=3.5 \mathrm{kV}, f_{\text {app }}=$ $750 \mathrm{~Hz}, H=2 \mathrm{~mm}$, and $Q=50 \mu \mathrm{L} / \mathrm{hr}$. As the duty cycle ratio $\rho$ increased from $30 \%$ to $50 \%$, the average line width enlarged from $22 \mu \mathrm{m}$ to $36 \mu \mathrm{m}$. When the duty cycle ratio $\rho$ was larger than $50 \%$, the average line width decreased from $36 \mu \mathrm{m}$ to $14 \mu \mathrm{m}$ while the duty cycle ratio increased from $50 \%$ to $80 \%$. The equivalent charge density and electrical field force reached the minimum value when duty cycle ratio $\rho$ was $50 \%$, such that the average line width reached its maximum value. 

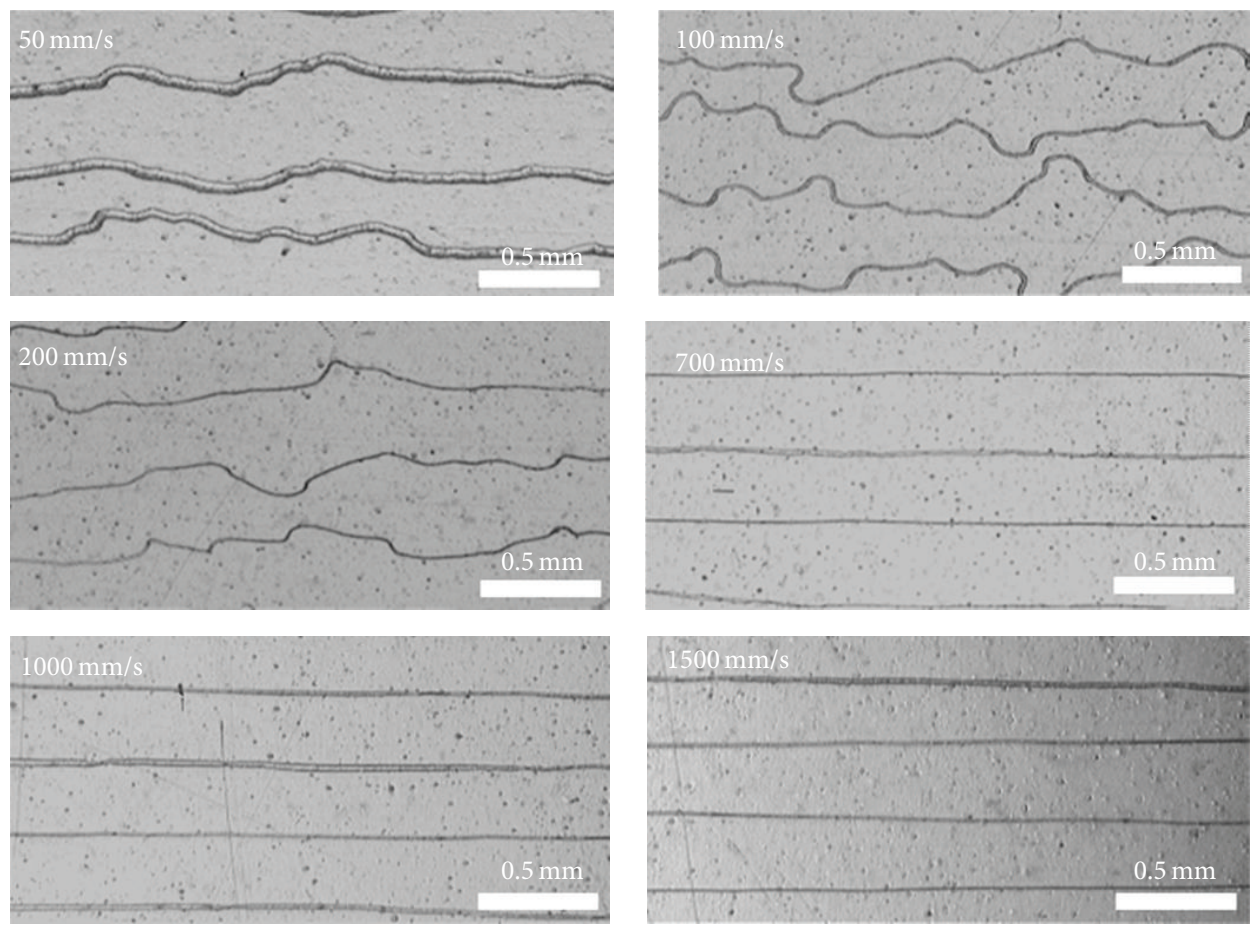

(a)

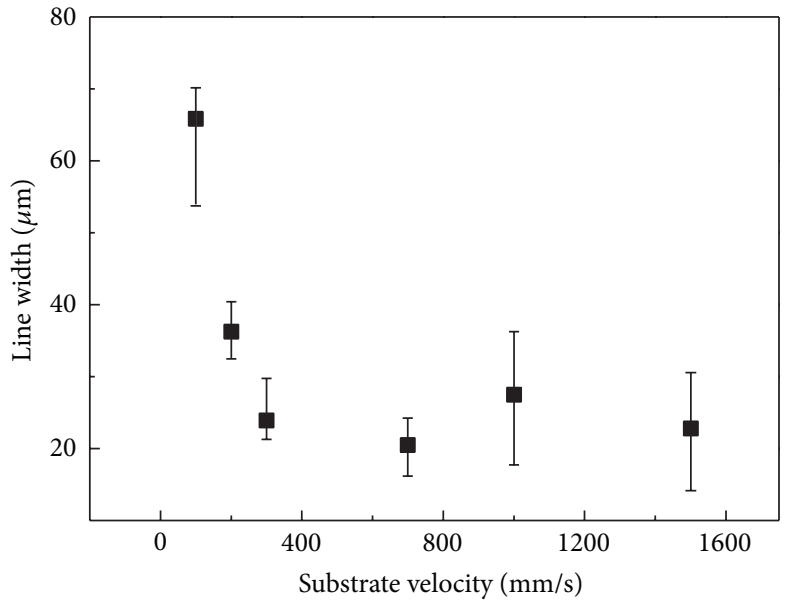

(b)

FIGURE 4: Micro/nanofibrous structure direct-written on insulate PET substrate under AC electrical field. The motion velocity of substrate was (a) $v_{\text {col }}=50 \mathrm{~mm} / \mathrm{s}$; (b) $v_{\text {col }}=100 \mathrm{~mm} / \mathrm{s}$; (c) $v_{\text {col }}=200 \mathrm{~mm} / \mathrm{s}$; (d) $v_{\text {col }}=700 \mathrm{~mm} / \mathrm{s}$; (e) $v_{\text {col }}=1000 \mathrm{~mm} / \mathrm{s}$; and (f) $v_{\text {col }}=1500 \mathrm{~mm} / \mathrm{s}$. Experimental condition: $C=14 \mathrm{wt} \%, U_{-}=-4 \mathrm{kV}, U_{+}=4 \mathrm{kV}, f_{\text {app }}=500 \mathrm{~Hz}, \rho=50 \%, H=2 \mathrm{~mm}$, and $Q=50 \mu \mathrm{L} / \mathrm{hr}$.

\section{Conclusion}

This paper reported an effective AC EDW approach to directwrite orderly micro/nanofibrous structures on the flexible insulating PET substrate. AC electrical field changed the charge transfer characteristics along the jet and decreased the Coulomb repulsive force applied on the following charged jet. And, then, injection stability of charged jet can be enhanced to build up stable straight jet under AC electrical field. With the help of stable straight jet under AC electrical field, parallel microfibrous structures can be direct-written on insulating
PET substrate. The minimum motion velocity of substrate required to direct-write straight line fibrous on insulating PET substrate was $700 \mathrm{~mm} / \mathrm{s}$. Line width of direct-written fibrous structure was in the range of $10 \mu \mathrm{m} \sim 40 \mu \mathrm{m}$. The line width reached its maximum value, when the negative voltage amplitude equals the positive ones $\left(U_{-}=U_{+}\right)$, AC voltage frequency $f_{\text {app }}=750 \mathrm{~Hz}$, and duty cycle ratio $\rho=50 \%$. This work provides an excellent way to increase the EDW controlling technology and accelerates the industrial application in the field of flexible electronic. 


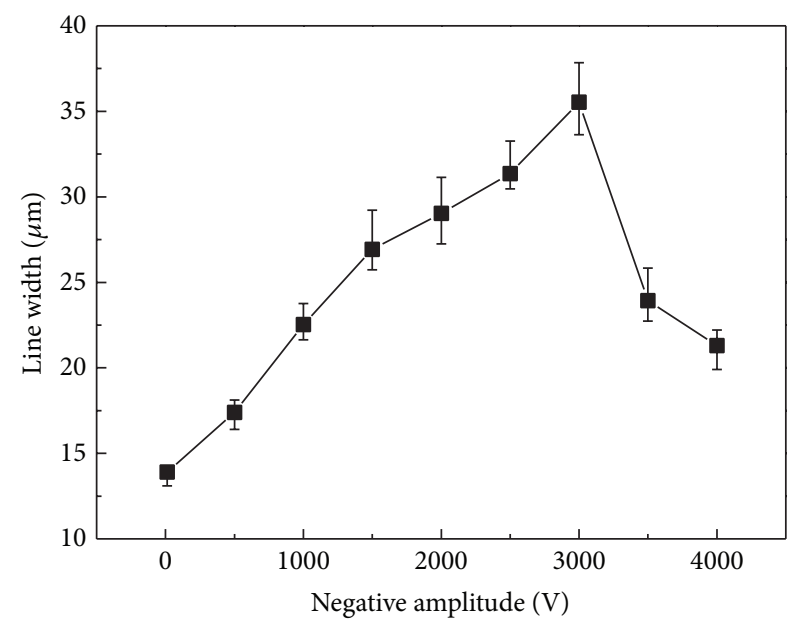

FIGURE 5: The relationship between line width and negative voltage amplitude. Experimental condition: $C=14 \mathrm{wt} \%, U_{+}=3 \mathrm{kV}, f_{\text {app }}=$ $750 \mathrm{~Hz}, \rho=50 \%, H=2 \mathrm{~mm}$, and $Q=50 \mu \mathrm{L} / \mathrm{hr}$.

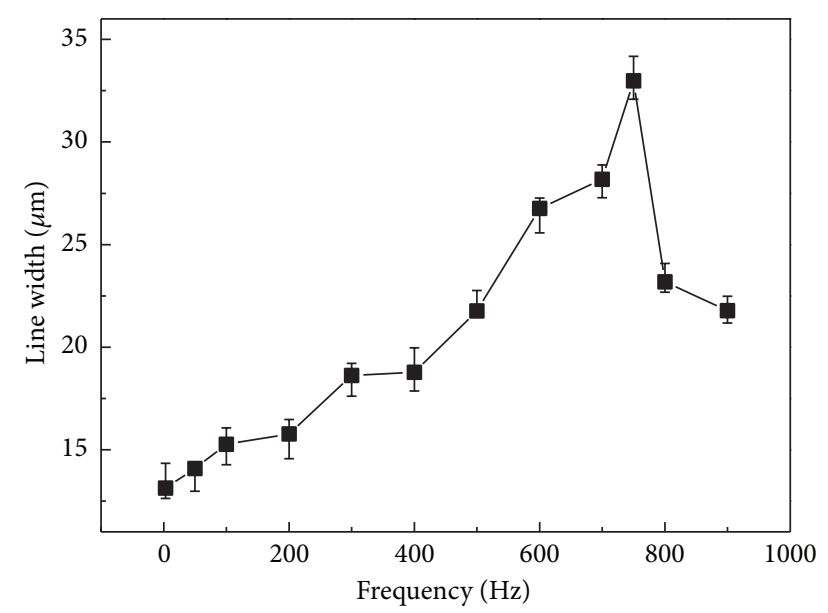

FIGURE 6: The relationship between line width and negative voltage amplitude. Experimental condition: $C=14 \mathrm{wt} \%, U_{-}=-1.5 \mathrm{kV}$, $U_{+}=3.5 \mathrm{kV}, \rho=50 \%, H=2 \mathrm{~mm}$, and $Q=50 \mu \mathrm{L} / \mathrm{hr}$.

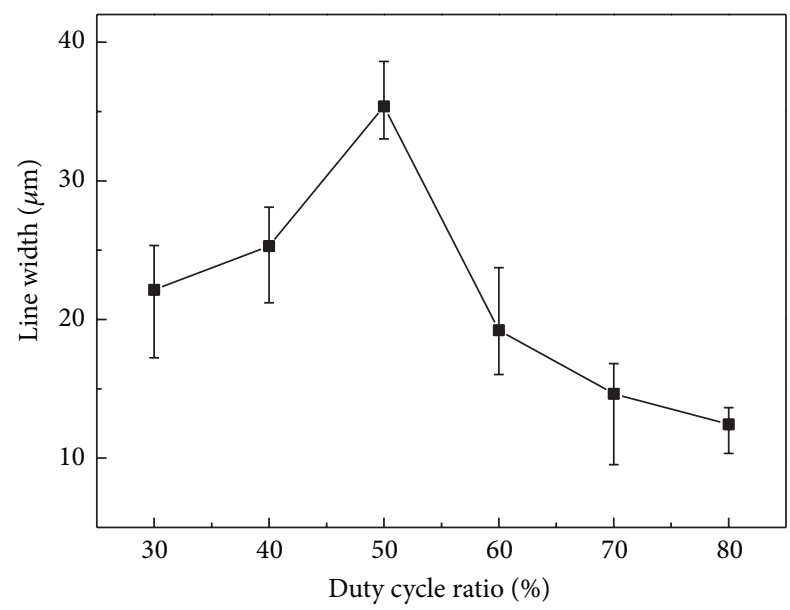

FIgURE 7: The relationship between line width and negative voltage amplitude. Experimental condition: $C=14 \mathrm{wt} \%, U_{-}=-1.5 \mathrm{kV}$, $U_{+}=3.5 \mathrm{kV}, f_{\text {app }}=750 \mathrm{~Hz}, H=2 \mathrm{~mm}$, and $Q=50 \mu \mathrm{L} / \mathrm{hr}$.

\section{Conflict of Interests}

The authors declare that there is no conflict of interests regarding the publication of this paper.

\section{Acknowledgments}

This work is supported by the National Natural Science Foundation of China (nos. 51305371 and 51305373), the Natural Science Foundation of Fujian Province (Grant no. 2013J05083), and the Ph.D. Programs Foundation of Ministry of Education of China (no. 20120121120035).

\section{References}

[1] J. Upadhyay, R. Das, S. Rao et al., "Manufacturing and characterisation of PMMA-graphene oxide (GO) nanocomposite sandwich films with electrospun nano-fibre core," Journal of Achievements in Materials and Manufacturing, vol. 55, no. 2, pp. 835-840, 2012.

[2] W. M. Choi, T. Tam, and N. B. Trung, "Fabrication of submicrometer graphene ribbon using electrospun nanofiber," Journal of Materials Science, vol. 49, no. 3, pp. 1240-1245, 2013.

[3] N. Zhu, W. Liu, M. Xue et al., "Graphene as a conductive additive to enhance the high-rate capabilities of electrospun $\mathrm{Li}_{4} \mathrm{Ti}_{5} \mathrm{O}_{12}$ for lithium-ion batteries," Electrochimica Acta, vol. 55, no. 20, pp. 5813-5818, 2010.

[4] Q. Wang, Y. Du, Q. Feng et al., "Nanostructures and surface nanomechanical properties of polyacrylonitrile/graphene oxide composite nanofibers by electrospinning," Journal of Applied Polymer Science, vol. 128, no. 2, pp. 1152-1157, 2013.

[5] Y. Zhang, Y. Wang, J. Jia, and J. Wang, "Nonenzymatic glucose sensor based on graphene oxide and electrospun $\mathrm{NiO}$ nanofibers," Sensors and Actuators B, vol. 171-172, pp. 580-587, 2012.

[6] Y.-L. Huang, A. Baji, H.-W. Tien et al., "Self-assembly of graphene onto electrospun polyamide 66 nanofibers as transparent conductive thin films," Nanotechnology, vol. 22, no. 47, Article ID 475603, 2011.

[7] H. Minemawari, T. Yamada, H. Matsui et al., "Inkjet printing of single-crystal films," Nature, vol. 475, no. 7356, pp. 364-367, 2011.

[8] S. H. Ko, H. Pan, C. P. Grigoropoulos, C. K. Luscombe, J. M. J. Fréchet, and D. Poulikakos, "All-inkjet-printed flexible electronics fabrication on a polymer substrate by low-temperature high-resolution selective laser sintering of metal nanoparticles," Nanotechnology, vol. 18, no. 34, Article ID 345202, 2007.

[9] D. Sun, C. Chang, S. Li, and L. Lin, "Near-field electrospinning," Nano Letters, vol. 6, no. 4, pp. 839-842, 2006.

[10] C. Chang, V. H. Tran, J. Wang, Y.-K. Fuh, and L. Lin, "Directwrite piezoelectric polymeric nanogenerator with high energy conversion efficiency," Nano Letters, vol. 10, no. 2, pp. 726-731, 2010.

[11] X. Wang, G. Zheng, G. He et al., "Electrohydrodynamic directwriting $\mathrm{ZnO}$ nanofibers for device applications," Materials Letters, vol. 109, pp. 58-61, 2013.

[12] I.-D. Kim, A. Rothschild, B. H. Lee, D. Y. Kim, S. M. Jo, and H. L. Tuller, "Ultrasensitive chemiresistors based on electrospun $\mathrm{TiO}_{2}$ nanofibers," Nano Letters, vol. 6, no. 9, pp. 2009-2013, 2006. 
[13] G. Zheng, W. Li, X. Wang, D. Wu, D. Sun, and L. Lin, "Precision deposition of a nanofibre by near-field electrospinning," Journal of Physics D, vol. 43, no. 41, Article ID 415501, 2010.

[14] H. Wang, G. F. Zheng, W. W. Li et al., "Direct-writing organic three-dimensional nanofibrous structure," Applied Physics A, vol. 102, no. 2, pp. 457-461, 2011.

[15] R. Kessick, J. Fenn, and G. Tepper, "The use of AC potentials in electrospraying and electrospinning processes," Polymer, vol. 45, no. 9, pp. 2981-2984, 2004.

[16] S. Ghashghaie, A. M. Bazargan, M. E. Ganji et al., "An investigation on the behavior of electrospun $\mathrm{ZnO}$ nanofibers under the application of low frequency AC electric fields," Journal of Materials Science: Materials in Electronics, vol. 22, no. 9, pp. 1303-1307, 2011.

[17] H. Mu, G. Zhang, S. Suzuki, and Y. Tanaka, "Surface charge distribution of polymeric insulating materials under HVAC," Proceedings of the Chinese Society of Electrical Engineering, vol. 30, no. 31, pp. 130-136, 2010.

[18] S. Sarkar, S. Deevi, and G. Tepper, "Biased AC electrospinning of aligned polymer nanofibers," Macromolecular Rapid Communications, vol. 28, no. 9, pp. 1034-1039, 2007.

[19] F. O. Ochanda, M. A. Samaha, H. V. Tafreshi, G. C. Tepper, and M. Gad-El-Hak, "Fabrication of superhydrophobic fiber coatings by DC-biased AC-electrospinning," Journal of Applied Polymer Science, vol. 123, no. 2, pp. 1112-1119, 2012.

[20] Y. Huang, L. Xu, G. Zheng, Z. Liu, and D. Sun, "Experiment on EHD printing under DC high voltage," Chinese Journal of Sensors and Actuators, vol. 23, no. 7, pp. 918-921, 2010.

[21] W.-W. Li, G.-F. Zheng, X. Wang, and D.-H. Sun, "Position deposition of electrospinning direct-writing nanofiber on pattern substrate," Optics and Precision Engineering, vol. 18, no. 10, pp. 2231-2238, 2010. 

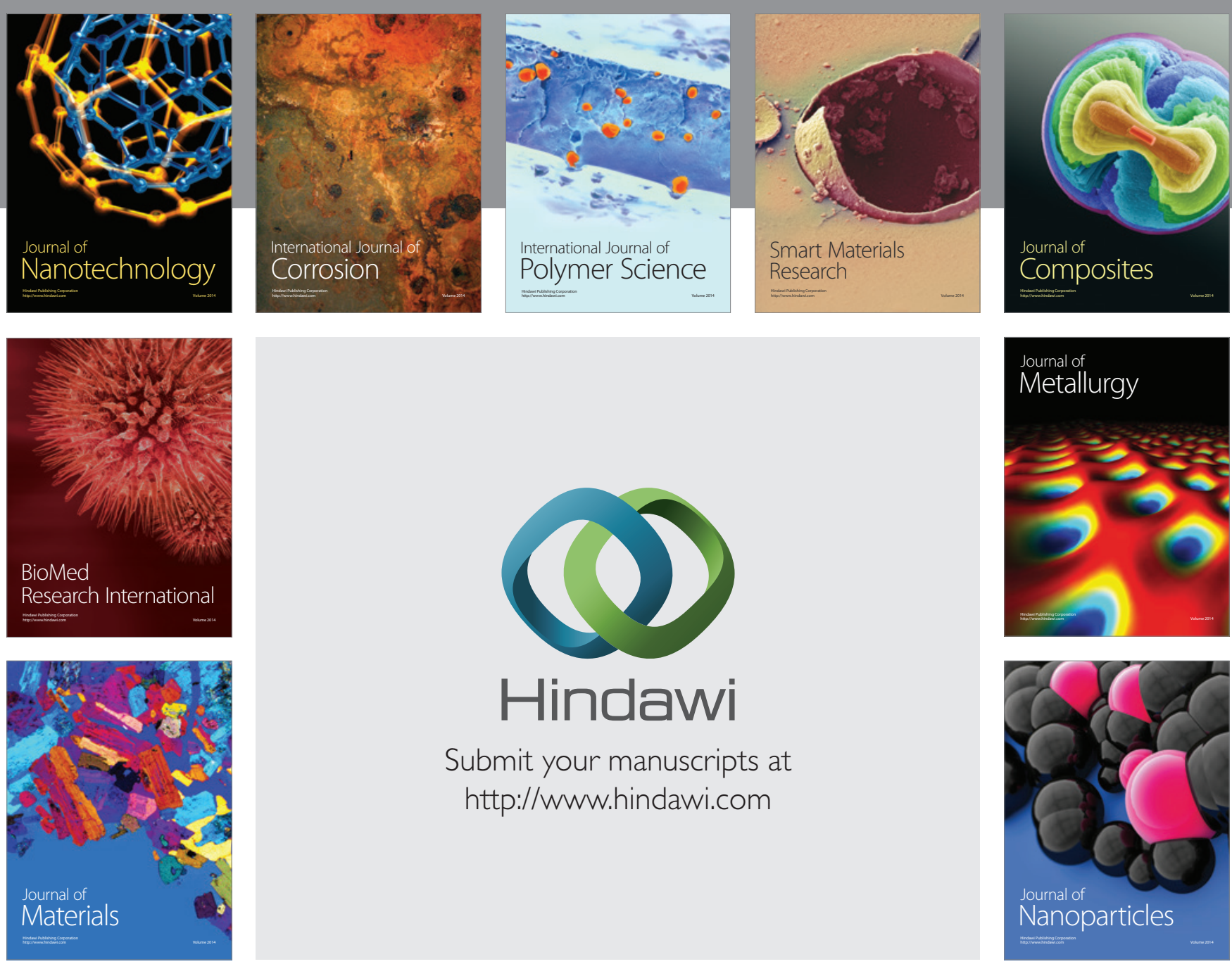

Submit your manuscripts at http://www.hindawi.com
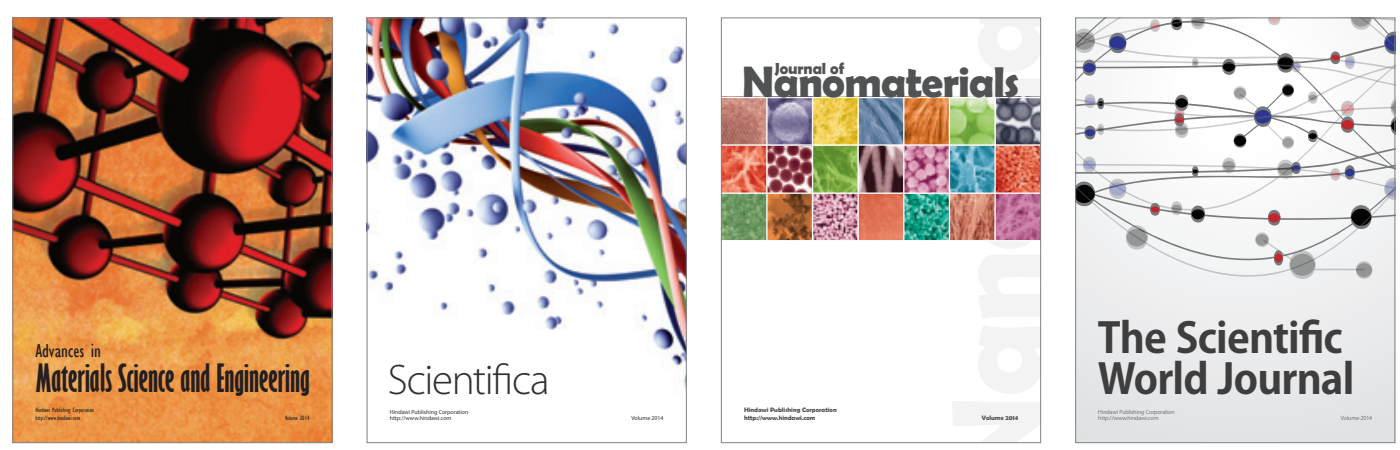

\section{The Scientific World Journal}
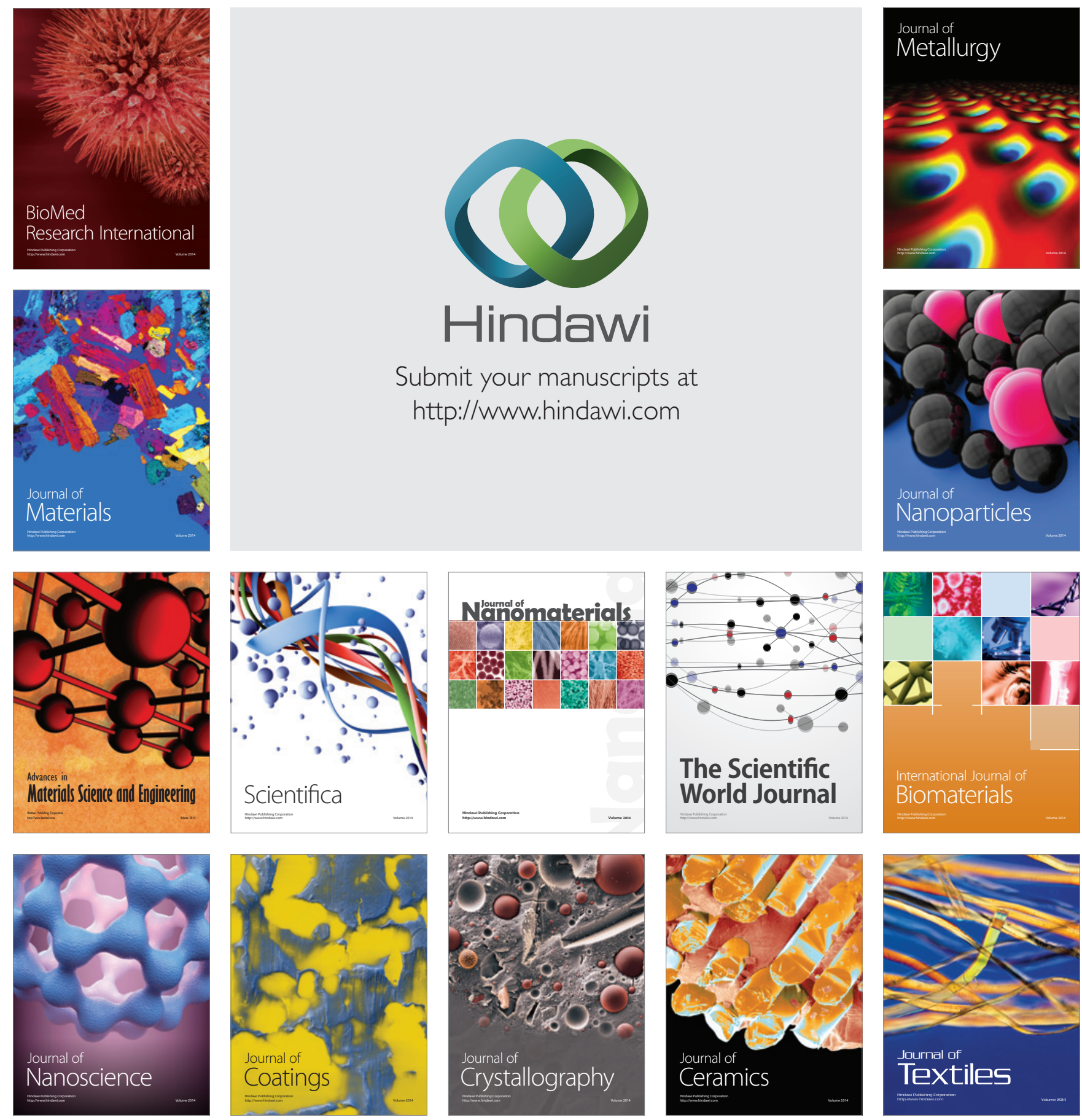